\title{
Schaftfrakturen am wachsenden Skelett (Kinder und Adoleszenten): Intramedulläre Stabilisierungsverfahren - State of the Art
}

\author{
C. Klemt, K.-J. Engler, F. Bonnaire, H. P. Friedl
}

\section{Zusammenfassung}

„Die stabile elastische Markraumschienung ist ein kindgerechtes, minimal traumatisierendes Osteosyntheseverfahren in der Versorgung metaund diaphysärer Extremitätenverletzungen. Bei richtiger Indikationsstellung und Technik stellt sie ein komplikationsarmes Verfahren dar und ist anderen Osteosyntheseverfahren und der konservativen Therapie überlegen.
Die vorliegende Arbeit gibt nach einem kurzen historischen Abriss, einer Beschreibung der biomechanischen Grundlagen und einer Darstellung der allgemeinen Operationstechnik einen Überblick über die heute verfügbaren Implantate sowie das jeweilige praktisch-technische Vorgehen am Ober- und Unterarm, Femur und Tibia.“

\section{Einleitung}

Die bisherige Domäne in der Behandlung von kindlichen Frakturen war die konservative Therapie. Sie stellt eine risikoarme Therapie dar, verbliebene Achsfehler unterliegen beim wachsenden Knochen einer gewissen Spontankorrektur. Schlechte funktionelle Ergebnisse bestimmter Frakturtypen sowie höhere Anforderungen an den Patientenkomfort mit funktioneller Nachbehandlung und der Entwicklung minimalinvasiver Osteosyntheseverfahren haben zu einem Umdenken geführt. So weiß man, dass nicht alle Achsfehler beim wachsenden Knochen spontan korrigiert werden. $\mathrm{Zu}$ nennen sind vor allem Rotationsfehler oder beispielsweise Valgusfehlstellungen des Unterschenkels.

Als Therapieziel in der Behandlung kindlicher Frakturen wird eine definitive Versorgung gefordert, welche eine schnellstmögliche Belastung und Wiederherstellung der Funktion zulässt mit einem Minimum an Aufwand [10].

Die Entwicklung der intramedullären elastischen Markraumschienung revolutionierte die Frakturbehandlung von kindli-

OP-JOURNAL 2000; 16: 188-193

(C) Georg Thieme Verlag Stuttgart · New York chen meta- und diaphysären Frakturen. Sie erfüllt alle wesentlichen Forderungen eines modernen Osteosyntheseverfahrens als kindgerechtes Verfahren, welches die besondere Biologie des wachsenden Knochens berücksichtigt. Die Methode ist mit geringem operativen Aufwand und minimalinvasiver Technik durchführbar ohne Tangieren der Wachstumsfugen. Sie erlaubt durch die elastische, stabile Osteosynthese in der Regel eine gipsfreie frühfunktionelle Nachbehandlung.

\section{Geschichtliche Entwicklung}

Schon gegen Ende des 19. Jahrhunderts wurden intramedulläre Osteosyntheseverfahren veröffentlicht, wobei überwiegend Elfenbeinstifte und -implantate zur Anwendung kamen. Niehans berichtete 1904 über die erste intramedulläre Schienung einer kindlichen Humerusfraktur mit Intramedullärnägeln. Verwendet wurden einfache Eisenlegierungen, welche zum Teil erhebliche Fremdkörperreaktionen verursachten oder Edelmetalle wie Gold und Silber mit einer allerdings ungenügenden Stabilität. So publizierte Schöne 1913 die Daten von 7 Patienten mit intramedullärer Schienung von Unterarmbrüchen durch verformbare Silberstäbe.

Erst die Entwicklung von Vanadiumstahl und V2a-Stahl ermöglichte die weitere Entwicklung der intramedullären Osteosynthese.

Wegbereiter für die moderne intramedulläre Osteosynthese waren die Brüder Rush in Amerika mit einer 1936 stabilisierten Monteggia-Fraktur und Küntscher in Deutschland, welcher 1939 die erste Marknagelung einer Femurschaftfraktur durchführte.

Es folgte die Bündelnagelung nach Hackethal, welches als Prinzip die stabile und formgerechte Markraumschienung mit einer Vielzahl von dünnen, elastischen Nägeln hatte.

Ender und Simon-Weidner entwickelten lange federnde Nägel zur Behandlung subtrochantärer Frakturen, über welche sie 1970 berichteten. Die ursprünglich für Erwachsene empfohlene Methode der Endernagelung wurde im weiteren Verlauf auf die kindlichen Frakturen erweitert.

Der Rumäne Firica veröffentlichte die Methode der elastischen stabilen Mark-

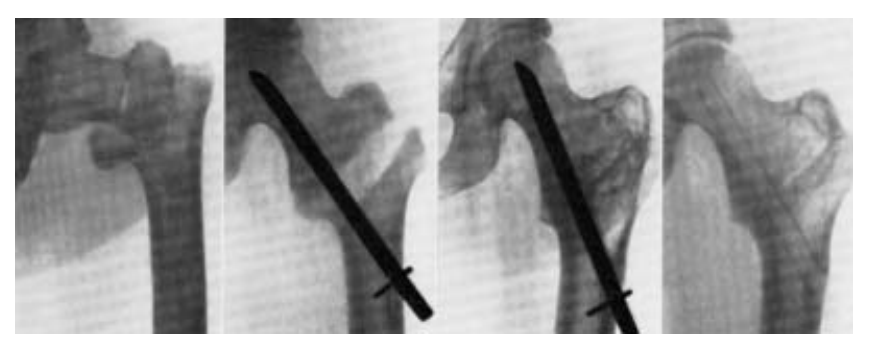

Abb. 1 Küntschernagelung einer pertrochantären Oberschenkelfraktur. 


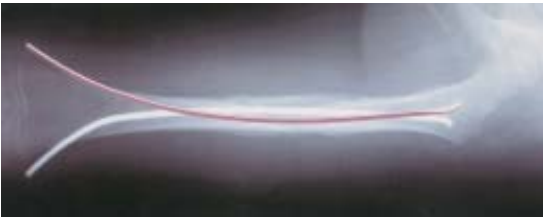

Abb.2 Prinzip der 3-Punkte-Abstützung.

raumschienung mit Hilfe des Instrumentariums von Ender. Es wurden mit dieser modifizierten Methode überwiegend erwachsene Patienten behandelt.

Von Metaizeau und Ligier wurde diese Idee aufgegriffen und nach experimentellen Vorarbeiten die intramedulläre elastische Markraumschienug zur Behandlung kindlicher Frakturen erweitert.

\section{Biomechanisches Prinzip}

Das Prinzip der intramedullären Markraumschienung stellt eine 3-Punkte-Abstützung zweier gegenläufiger Kraftträger dar. Jeder dieser Kraftträger stützt sich in der Kortikalis des Eintrittpunktes, der Kortikalis in Frakturhöhe und im spongiösen Knochen des Gegenfragmentes $a b$.

Die Elastizität und Aufspannung der Implantate bewirkt eine Stabilität und Rück- stellkraft in allen räumlichen Ebenen. Sowohl Schub, Rotation und Kompression werden neutralisiert und es resultieren lediglich Mikrobewegungen im Frakturbereich.

Diese Mikrobewegungen bewirken einen Stimulus zur Kallusbildung und erlauben eine schnelle Frakturheilung.

Die zu erwartende Wachstumsstimulation ist bei der elastischen Markraumschienung gegenüber der konservativen Behandlung oder der Plattenosteosynthese am geringsten. Sie bewirkt durch die Mikrobewegungen zwar eine ausgeprägte lokale Stimulation, aber nur eine sehr geringe Stimulation der Wachstumsfugen.

Die Y-förmige Verstrebung vermindert nach Firica die notwendige Tragefähigkeit des Implantats zur Kompensation einer einwirkenden Kraft um 30\% [14].

\section{Operationstechnik}

Die zu versorgende Extremität soll frei beweglich abgedeckt werden, bei Verletzungen der oberen Extremität wird der Arm auf einem Handtisch ausgelagert. Der C-Bogen muss so plaziert sein, dass Rö-Kontrollen in 2 Ebenen problemlos sind.
Vor der Operation wird die erforderliche Schienenstärke bestimmt. Sie beträgt ein Drittel des Markraumdurchmessers an seiner schmalsten Stelle. An Radius und Ulna wählt man eine Dimension, welche zwei Drittel des Markraumdurchmessers entsprechen.

Grundsätzlich ist die Epiphyse und Epiphysenfuge zu schonen. Mit Hilfe des Bildwandlers gelingt hier die sichere Bestimmung der Eintrittsstelle. Die Markraumeröffnung wird mit einem Bohrer und/oder einem Pfriem durchgeführt. Der Eintrittswinkel beträgt dabei $30^{\circ}-$ $45^{\circ}$. Das Eröffnungsloch sollte so erweitert werden, dass die Schiene ohne Widerstand im Bereich der Eintrittsstelle gleiten kann.

Werden die beiden Schienen von gegenüberliegenden Stellen (medial und lateral des frakturierten Knochens) eingebracht, ist auf die gleiche Höhe der Eintrittsstellen zu achten, da eine asymmetrische Implantation zwei unterschiedliche Spannungsbogen verursacht und so eine Fehlstellung resultiert.

Bei gleichseitiger Implantation sollten zwei Bohrlöcher angelegt werden. Dabei wird die frakturfern eingebrachte Schiene nach Erreichen der Diaphyse um $180^{\circ}$ gedreht, damit sie in einem S-
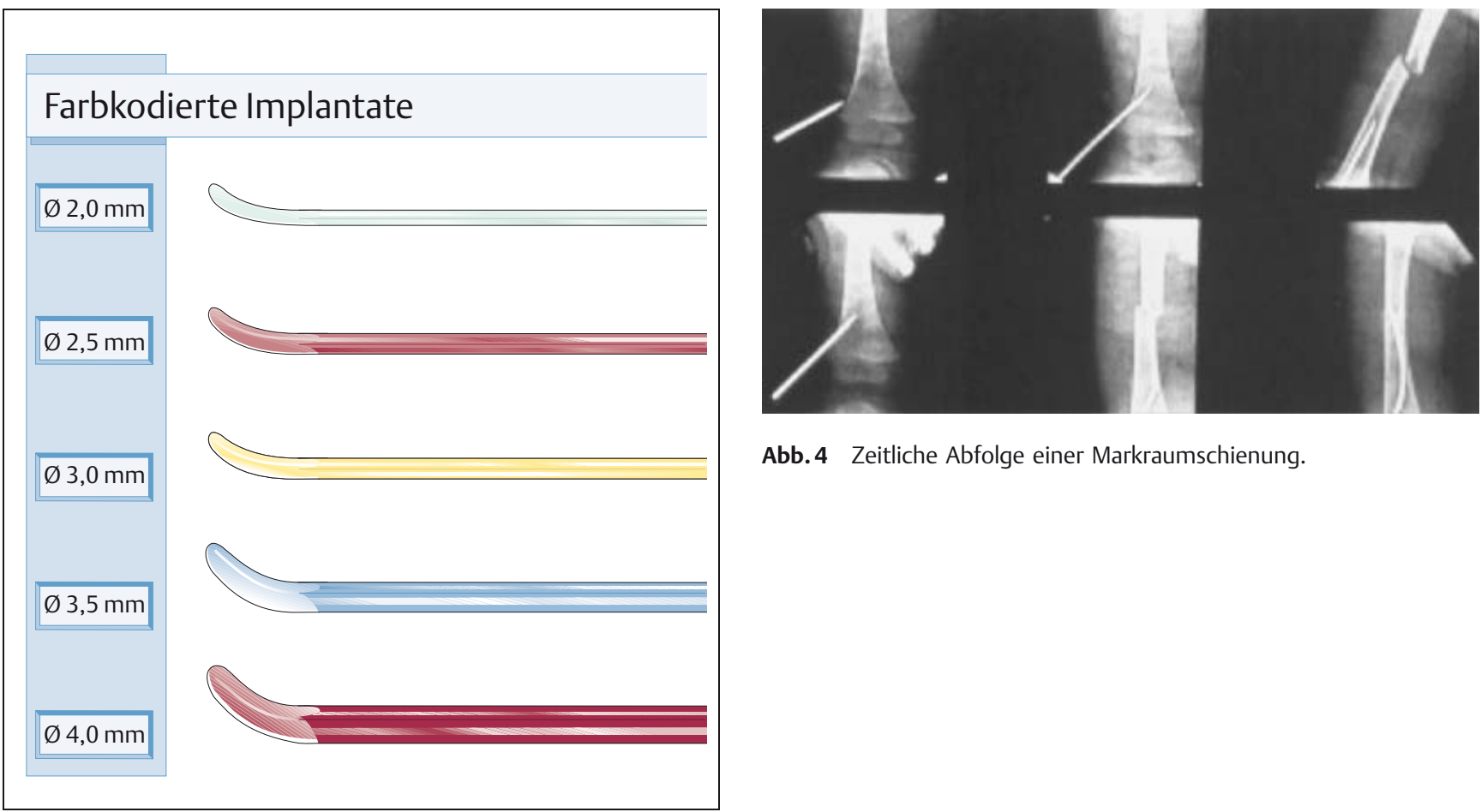

Abb.4 Zeitliche Abfolge einer Markraumschienung.

Abb.3 Implantate in verschiedenen Größen. 
förmigen Verlauf in der diagonal entgegengesetzten metaphysären Spongiosa verankert werden kann.

Ob eine aszendierende oder deszendierende Implantatrichtung gewählt wird, hängt im Wesentlichen von der Frakturlokalisation ab. Kommt die Eintrittsstelle zu nahe an der Bruchstelle zu liegen, kann das Fragment durch die Schienen nicht ausreichend geführt, stabilisiert oder eine achsengerechte Ausrichtung erzielt werden.

Bei Schrägfrakturen wird zuerst diejenige Schiene eingebracht, welche mit ihrer Spitze senkrecht auf die Frakturlinie trifft. Bei Torsionsfrakturen wird empfohlen, die Implantation auf der Seite der langen Kortikalis zu beginnen. Drehfehler können durch Drehen der Schienen korrigiert werden.

Die Implantation der Schienen erfolgt manuell durch leichte Drehbewegungen. Das Vorantreiben der Marknägel darf auf keinen Fall durch Hammerschläge erfolgen, da das Ausbrechen der Gegenkortikalis droht und eine suffiziente Abstützung zunichte gemacht wird. Lediglich die Verankerung der Nägel im spongiösen Knochen des Gegenfragmentes wird mit vorsichtigen Schlägen ausgeführt. Dabei ist unbedingt darauf zu achten, dass die Wachstumsfuge nicht kompromitiert wird.

Wenn nach 3-4 Versuchen keine Auffädelung der Hauptfragmente gelingt, kann eine Interposition z.B. von Muskulatur vorliegen, was die offene Reposition über eine kleine Inzision in Höhe der Fraktur erforderlich macht.

Neben einer asymmetrischen Schienenimplantation, bergen zu kurze oder zu dünne Implantate weitere Fehlerquellen.

$\mathrm{Zu}$ vermeiden sind außerdem umeinander gewundene Schienen da eine Aufspannung und die erforderliche 3Punkte-Abstützung dadurch verhindert wird.

\section{Obere Extremität}

\section{Humerus}

Eine Osteosynthese ist grundsätzlich nur erforderlich, wenn die Reposition und Retention nicht oder nur ungenügend gelingt. In der Regel heilen Humerusschaftfrakturen unter konservativer Therapie schnell.

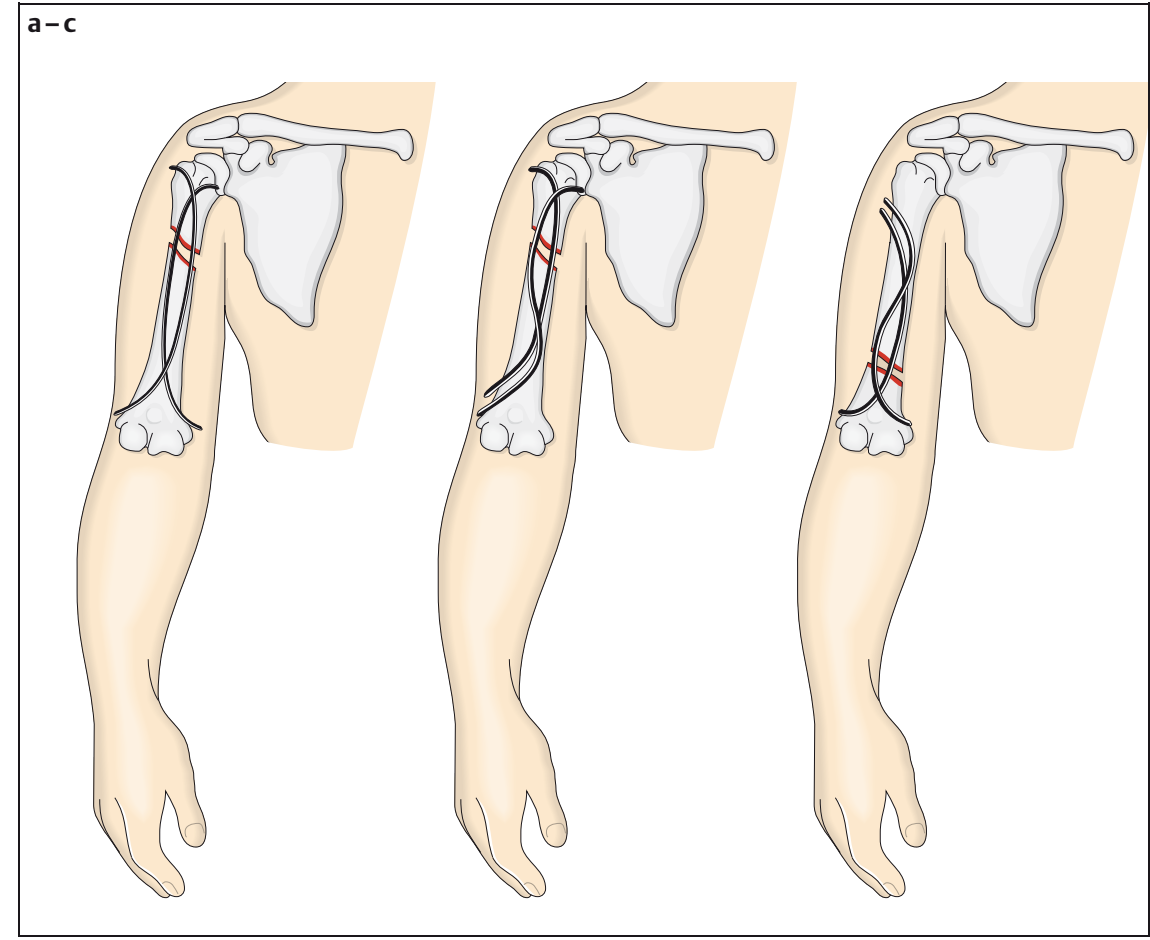

Abb.5 Elastische Markraumschienung am Humerus. (a) aszendierende Schienung über einen radialen Zugang. (b) aszendierende Schienung von radial und ulnar. (c) deszendierende Schienung.

Eine erweiterte Indikation stellen das Polytrauma, Gefäß-/Nervenverletzungen oder beidseitige Verletzungen dar.

Indikationen zur intramedullären Osteosynthese am proximalen Oberarm und Oberarmschaft sind nach der AO-Klassifikation: A1-A3-, B1-B3- und C2-Frakturen.

In den meisten Fällen kann eine aszendierende Implantation durchgeführt werden. Die Inzisionen werden beidseits am jeweiligen Epikondylus ca. $1-2 \mathrm{~cm}$ lang gesetzt und der Markraum mit dem Bohrer in $45^{\circ}$ eröffnet und gegebenenfalls mittels Pfriem erweitert. Zur Vermeidung einer Verletzung des N. ulnaris sollte die Kortikalis medialseitig im anterioren Bereich perforiert werden. Alternativ bietet sich die distale einseitige radiale Implantation über zwei Bohrlöcher an.

Bei Frakturen im distalen Drittel der Diaphyse ist die deszendierende Implantation erforderlich, diese erfolgt über einen Zugang distal des Ansatzes des M. deltoideus. Es wird eine etwa $3 \mathrm{~cm}$ lange Hautinzision durchgeführt und der M. brachialis bis auf den Knochen gespalten. In einem Abstand von $3 \mathrm{~cm}$ werden die Eintrittslöcher gebohrt.
Die Nachbehandlung erfolgt im Gilchristverband bis Schmerzfreiheit erreicht ist. Nach wenigen Tagen kann funktionell weiterbehandelt werden. Eine Röntgenkontrolle ist postoperativ, sowie nach 3 Wochen erforderlich. Die Entfernung der Implantate kann nach 3-4 Monaten geplant werden.

\section{Unterarm}

Nur in sehr seltenen Fällen müssen isolierte Radius- oder Ulnafrakturen operativ versorgt werden. Eine Ausnahme stellt dabei die Monteggia-Fraktur (AO-Klassifikation A1.3) dar. Weitere Indikationen sind die A3-Frakturen, alle B-Frakturen sowie die 2-Etagen-Frakturen der CGruppe.

Die Standardversorgungen am Unterarm sind die aszendierende Radiusschienung und die deszendierende Ulnaschienung.

Die Hautinzision erfolgt für den Radius in Höhe des Proc. styloideus nach proximal mit $2-3 \mathrm{~cm}$ Länge. Um eine Verletzung des R. superficialis des N. radialis auszuschließen, ist dieser vor der Markraumeröffnung darzustellen. 

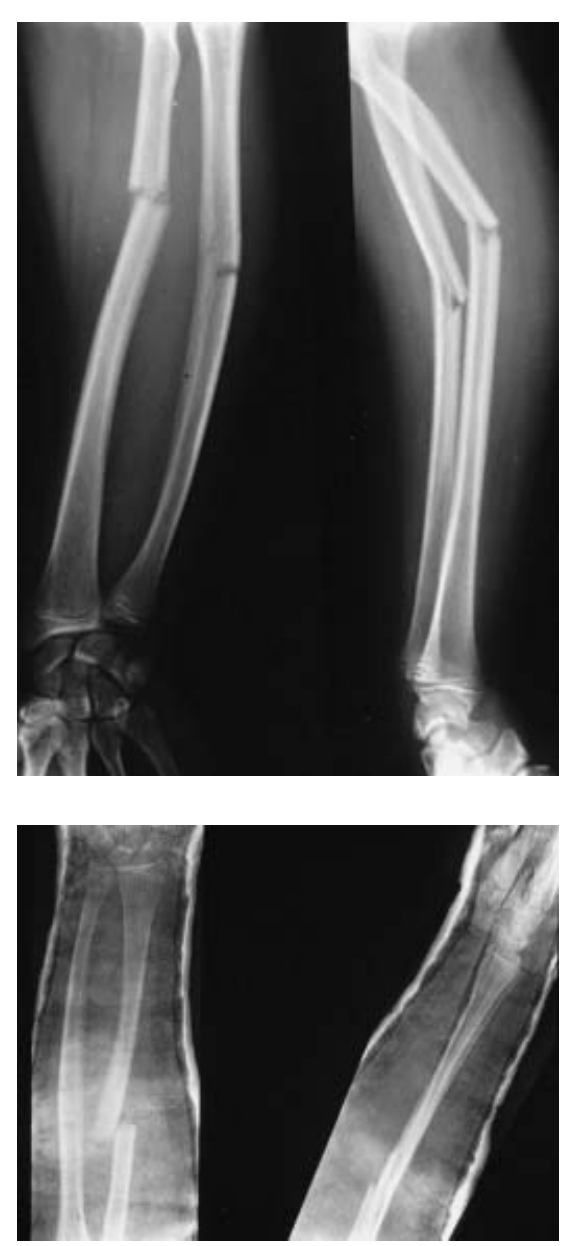

Abb. 6 Unterarmschaftfraktur, nach Reposition im Gips nicht retinierbar.

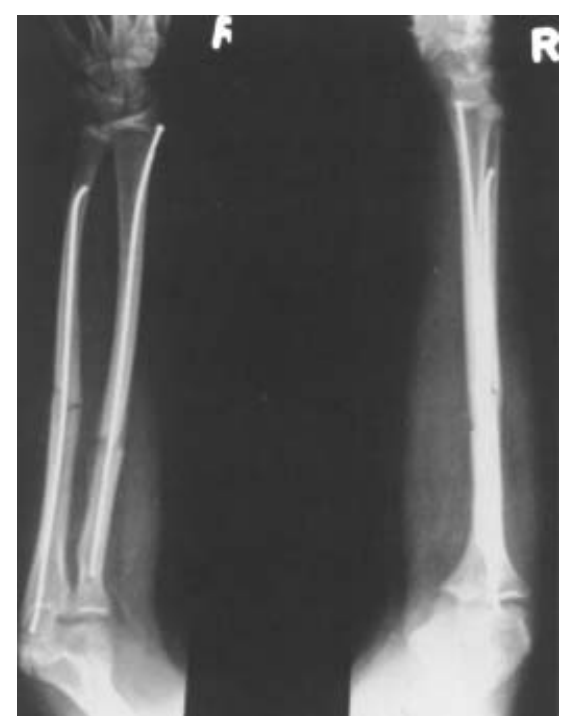

Abb.7 Achsengerechte Stellung nach Osteosynthese mit Markraumschienen.

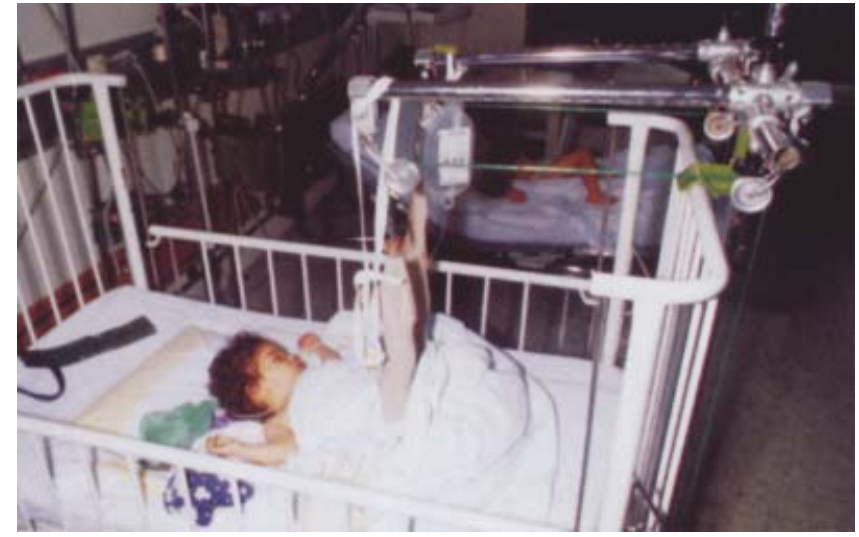

Abb. 8 Overheadextension einer Femurschaftfraktur beim einjährigen Kind.
Die Eröffnung erfolgt in $30^{\circ}-45^{\circ}$ mit dem Bohrer oder Pfriem.

Die deszendierende Schienung der Ulna erfolgt über eine dorso-radiale Inzision etwa 4-5 cm distal der Spitze des Olekranons. Es werden damit Verletzungen oder Irritationen des N. ulnaris z. B. durch das überstehende Nagelende vermieden.

Über die divergierenden Schienen in Radius und Ulna gelingt ein Aufspannen der Membrana interossea, was somit zu einer weiteren Stabilisierung beiträgt. Drehfehler können durch Drehen der Nägel ausgeglichen werden. Kleine Achsfehler oder Seitverschiebungen um Kortikalisbreite sind unbedeutend für das postoperative Ergebnis.

Radiushalsfrakturen werden entsprechend dem Standardzugang für Radiusschaftfrakturen aszendierend versorgt. Unter Bildwandlerkontrolle wird dabei das Radiusköpfchen in die lateralste Position eingestellt und die Spitze der Markraumschiene entsprechend ausgerichtet. Unter Druck mit dem Daumen auf das Radiusköpfchen wird der Nagel bis in den unteren Abschnitt der Epiphyse vorgetrieben. Anschließend wird die Schiene um $180^{\circ}$ gedreht, bis die Spitze nach medial zeigt. Durch diese Drehung gelingt die Reposition des Radiusköpfchens. Eine Überkorrektur nach medial wird durch die Spannung des lateralen Periost vermieden.

Die Nachbehandlung der Unterarmfrakturen erfolgt funktionell, eine postoperative Ruhigstellung ist nicht erforderlich. Die Entfernung der Implantate kann nach 3-4 Monaten erfolgen.

\section{Untere Extremität}

Femur

Bei allen dislozierten Oberschenkelfrakturen jenseits des 4. Lebensjahres besteht prinzipiell die Indikation zur primären, definitiven operativen Versorgung. Bei jüngeren Kindern ist eine konservative Therapie z.B. durch Overheadextension oder Becken-Bein-Gips angezeigt.

Indikationen für die intramedulläre Markraumschienung sind im mittleren Schaftbereich die Typen A1-A3, B1-B3 und $\mathrm{C} 2$. Relative Indikationen stellen die subtrochantären und distalen metaphysären Frakturen dar.

Die Standardversorgung erfolgt als aszendierende Osteosynthese über Hautinzisionen medial und lateral der Femurkondyle von der kranialen Patellaspitze etwa $3 \mathrm{~cm}$ nach proximal. Wichtig ist eine ausreichende Spaltung der Faszie, um spätere Irritationen durch die gekürzten Nagelenden und der damit verbundenen schmerzhaften Bewegungseinschränkung im Kniegelenk vorzubeugen. Die Eintrittstellen sollen 1-2 cm proximal der Epiphysenfugen liegen. Der Eintrittswinkel beträgt etwa $45^{\circ}$. Die Reposition wird unter Durchleuchtungskontrolle in zwei Ebenen durchgeführt, gegebenenfalls kann eine Schanz-Schraube im distalen Fragment als Re-positionshilfe verwendet werden.

Nach exakter Reposition werden die Spitzen der Schienen in der festen Spongiosa subtrochantär und im medialen Schenkelhals verankert.

Bei der Reposition ist auf die korrekte Rotation zu achten und eine Distraktion sollte vermieden werden. 

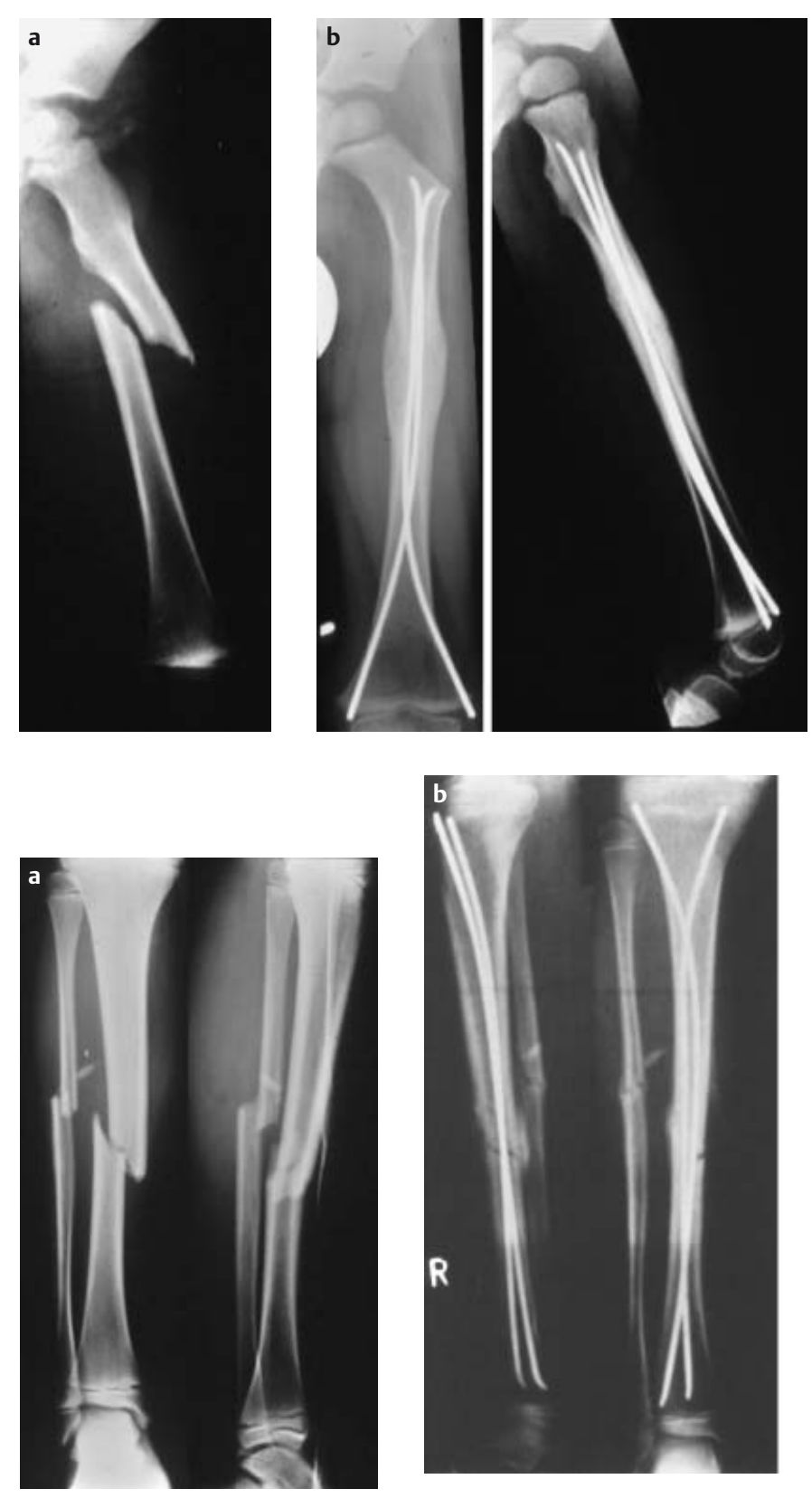
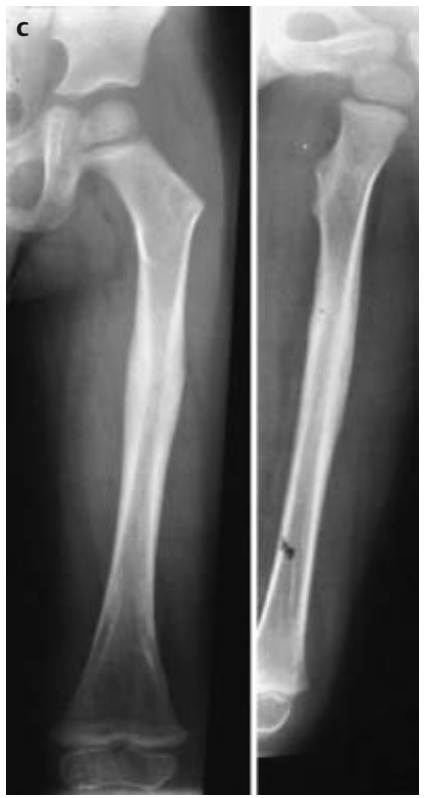

Abb. 93 Jahre altes Kind mit Femurschaftfraktur, Osteosynthese mit intramedullärer Markraumschienung und Ausheilungsergebnis.

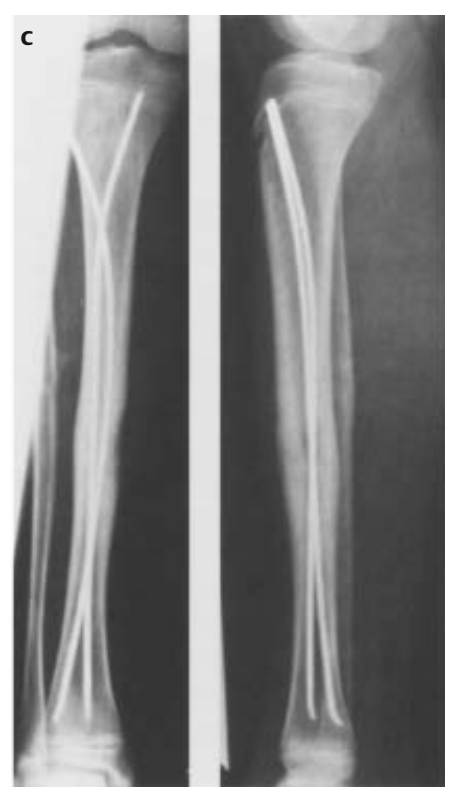

Abb.10 Unterschenkelschaftfraktur mit Versorgungs- und Ausheilungsergebnis.
In der Behandlung von Frakturen im distalen Drittel ist der Zugang von lateral subtrochatär als deszendierende Implantation zu wählen. Es werden zwei separate Bohrlöcher im Abstand von 2-3 cm für den Eintritt der Marknägel vorbereitet.

Die elastische Markraumschienung von Femurfrakturen ist in der Regel übungsstabil. Querfrakturen können dabei ab der zweiten Woche teilbelastet werden. Lange Schräg- und Torsionsbrüche sind nicht belastungsstabil und bedürfen einer Entlastung für 3-4 Wochen.
Die Entfernung der Implantate erfolgt nach 4-6 Monaten.

\section{Tibia}

Eine Indikation zur intramedullären Osteosynthese von Unterschenkelfrakturen ist nur selten gegeben. Sie wird durchgeführt bei Frakturen, bei denen der konservative Behandlungsversuch mißlingt. Es ist auf Rotations- und Valgusfehlstellungen zu achten, da diese nicht oder nur inkomplett im Laufe des Wachstums korrigiert werden.
Die Frakturtypen, welche sich für die intramedulläre Schienung eignen, sind für die Schaftfrakturen alle A- und B-Typen sowie C2-Frakturen. Außerdem Unterschenkelfrakturen vom Typ 41 A2 und $43 \mathrm{~A} 1$.

Die Schienung erfolgt deszendierend mit Hautinzisionen medial- und lateralseitig des Tibiakopfes distal der Tuberositas tibiae. Der Markraum wird in einem Winkel von $45^{\circ}-60^{\circ}$ eröffnet. 
$\mathrm{Zu}$ beachten ist, dass eine Distraktion der Fraktur vermieden wird und die Spitzen der Schienen nach dorsal zeigen, um die physiologische Antekurvation wiederherzustellen.

Bei stabiler Versorgung erfolgt die Nachbehandlung funktionell mit Entlastung an Gehstöcken für 3 Wochen. In Ausnahmen kann eine passagere Gipsbehandlung für 2 Wochen erforderlich sein.

Die Entfernung der Implantate erfolgt nach 4-6 Monaten.

\section{Schlussfolgerung}

Die stabile elastische Markraumschienung ist ein kindgerechtes, minimal traumatisierendes Osteosyntheseverfahren in der Versorgung meta- und diaphysärer Extremitätenverletzungen. Bei richtiger Indikationsstellung und Technik stellt sie ein komplikationsarmes Verfahren dar und ist anderen Osteosyntheseverfahren und der konservativen Therapie überlegen.

\section{Literatur}

${ }^{1}$ Illgen R, Rodgers WB, Hresko M, Waters P, Zurakowski D, Kasser J. Femur Fractures in Children: Treatment with Early Sitting Spica Casting. J Pediatr Orthop Vol 18, 1998; 4 : 481-487

${ }^{2}$ Ligier J, Metaizeau J, Prevot J, Lascombes P. Elastic Stable Intramedullary Nailing of Femoral Shaft Fractures in Children. J Bone Joint Surg. 70-B, 1988; 1: 74-77

3 Ostermann P, Richter D, Mecklenburg K, Ekkernkamp A, Muhr G, Hahn M. Unterarmfrakturen im Kindesalter. Unfallchirurg 1999; 102: 784-790

${ }^{4}$ Carey TP, Galpin RD. Flexible Intramedullary Nail Fixation of Pediatric Femoral Fractures. Clin Orthop Rel Res 1996; 332: 110-118

${ }^{5}$ Richter D, Peter A, Ostermann P, Ekkernkamp A, Muhr G, Hahn M. Elastic Intramedullary Nailing: A Minimally Invasive Concept in the Treatment of Unstable Forearm Fractures in Children. J Peditr Orthop

${ }^{6}$ Vol 18, 1998; 4: 457-461

7 Oestern H, Rieger G, Jansen Th. Intramedulläre Osteosynthesen beim Kind. Unfallchirurg 2000; 103: 2-11

8 Oetiker J, Komorek W, Meyer R, Kappeler U. Die Prevot-Nagelung, eine elegante Methode. Unfallchirurg 1996; 99: 327-331

${ }^{9}$ Dietz H, Schmittenbecher P, Illing P. Intramedulläre Osteosynthese im Wachstumsalter. Urban \& Schwarzenberg. München, Wien, Baltimore 1997

${ }^{10}$ Weinberg A, Reilmann H. Frakturen der Diaphyse im Kindesalter. Untere Extremität Unfallchirurg 2000; 102: 132-140
${ }^{11}$ Weinberg A, Reilmann H. Frakturen der Diaphyse im Kindesalter. Obere Extremität. Unfallchirurg 2000; 103: 144-155

${ }^{12}$ Lascombes P, Prevot J, Ligier J, Metaizeau J, Poncelet T. Elastic Stable Intramedullary Nailing in Forearm Shaft Fractures in Children: 85 Cases. J Pediatr Orthop Vol. 10, 1990; 2: $167-171$

${ }^{13}$ Huber R, Keller H, Huber P, Rehm K. Flexible Intramedullary Nailing as Fracture Treatment in Children. J Pediatr Orthop 1996; 16: $602-605$

${ }^{14}$ Firica A, Popescu R, Scarlet M. L'Osteosynthese Stable Elastique, Nouveau Concept Biomechanique. Etude Experimentale. Rev Chir Orthop 1981; 67: 82-91

\section{Dr. med. Ch. Klemt \\ Oberarzt \\ Dr. med. K. J. Engler \\ Assistenzarzt}

Priv.-Doz. Dr. med. F. Bonnaire

Leitender Oberarzt

Prof. Dr. med. H. P. Friedl

Ärztlicher Direktor

Chirurgische Universitätsklinik Abteilung Unfallchirurgie

Hugstetterstr. 55

79106 Freiburg 